\title{
Detection \& Identification of Tumor Region from MRI Brain Image using Image Segmentation
} \author{
Padmani S. Judape ${ }^{*}$, Prof. Pragati Patil2 ${ }^{2}$ Prof. Gajanan Patle ${ }^{3}$ \\ ${ }^{* 1}$ M Tech Student, Department of Computer Science and Engineering, Abha Gaikwad-Patil College of Engineering \& Technology, Nagpur, \\ India \\ ${ }^{2}$ Assistant Professor, Department of Computer Science and Engineering, Abha Gaikwad-Patil College of Engineering \& Technology, \\ Nagpur, India \\ ${ }^{3}$ Assistant Professor, Department of Computer Science and Engineering, Abha Gaikwad-Patil College of Engineering \& Technology, Nagpur, \\ India
}

\begin{abstract}
Brain tumor detection and segmentation is one in every of the foremost difficult and time overwhelming task in medical image process. Magnetic resonance imaging (MRI) may be a medical technique, in the main utilized by the radiotherapist for visualization of internal structure of the body with none surgery. Magnetic resonance imaging provides plentiful info regarding the human soft tissue that helps within the designation of neoplasm (brain tumor). Correct segmentation of MRI image is very important for the designation of brain tumor by laptop motor-assisted clinical tool. When acceptable segmentation of brain man pictures, growth is assessed to malignant and benign, that may be a troublesome task because of complexness and variation in growth tissue characteristics like its form, size, grey level intensities and site. Taking in to account the said challenges, this analysis is concentrated towards highlight the strength and limitations of earlier projected classification techniques mentioned within the up to date literature. Besides summarizing the literature, the paper additionally provides an important analysis of the surveyed literature that reveals new sides of analysis.
\end{abstract}

Keywords : Brain Tumor Detection, Magnetic Resonance Imaging

\section{INTRODUCTION}

Brain tumor is one of the prime causes behind the increase in mortality among people. A tumor is an abnormal growth caused by cells reproducing themselves in an uncontrolled manner. Brain tumor is a debilitating condition and is one of the leading causes of cancer -related deaths in the world today. Depending on the direction in which the tumor is spreading, the brain tumor is classified into various categories and the course of action for its treatment is chosen. Usually tumor contains more water as well as more Hydrogen atoms than normal tissue. So the intensity of tumor regions in MRI images will be different from normal tissues. The effects of a brain tumor are dependent on the size and location of the tumor and how much it has spread. There are two types of brain tumor: malignant and benign. Malignant, or cancerous, tumors often invade surrounding tissue and can spread to other parts of the body through the blood stream or lymphatic system. They can also erode 'healthy' tissue, as the cells that make up a malignant tumor share very little in common with the healthy cells that surround them. Because malignant tumors often grow and spread rapidly, early diagnosis can increase the chances of survival. If caught early, they will have had less chance to destroy healthy brain tissue, and are less likely to have spread to other parts of the body. Sometimes, brain tumors are the result of other 
malignant tumors' in other parts of the body that have spread to the brain - these are known as 'metastases', or 'secondary tumor. These are always malignant. Benign, or non-cancerous, tumors tend to grow more slowly and do not spread, although people can have more than one benign tumor. A benign brain tumor can put pressure on the brain as it grows inside the enclosed space of the skull, and this may compress and damage healthy tissue. Magnetic Resonance Imaging is one of the best technologies currently being used for detecting Brain Tumor at both early and advanced stages. Modern imaging techniques, such as MRI and CT scans, have made it possible for doctors to accurately judge the size and location of a tumor, making surgery possible where the growth is accessible, and allowing them to estimate how treatable it is. Surgery to remove all or part of a brain tumor is called a craniotomy, where the skull is opened. Where the entire tumor cannot be removed, radiotherapy or anti-cancer drugs may be tried to slow, limit or stop its spread. Brain tumor segmentation for MR images is a difficult and challenging task due to variation in type, size, location and shape of tumors. Real time diagnosis of tumors by using more reliable algorithms has been the main focus of the latest developments in medical imaging and detection of brain tumor in MR images and CT scan images has been an active research area. The separation of the cells and their nuclei from the rest of the image content is one of the main problems faced by most of the medical imagery diagnosis systems. The process of separation i.e. segmentation, is paid at most importance in the construction of a robust and effective diagnosis system. Images Segmentation is performed on the input images. This enables easier analysis of the image thereby leading to better tumor detection efficiency.

\section{OBJECTIVES}

- To collect/acquire the CT scan images from normal subjects and patients with Brain/Head injury.

- To evaluate changes in different TBI parameters in and compare them with normal subjects.

- To develop algorithms to extract features from CT scan Images.

- To develop an automatic classifier for diagnosis based on above parameters using Proposed Clustering method.

\section{SCOPE}

The technique proposed will be helpful in extracting the tumor portion; it has provided an accurate demarcation of the boundary of the tumor, along with correct visual location of the tumor with the help of a bounding circle. This work will also provide a diagnosis decision whether the tumor is present or absent along with the exact size of the tumor. This decision can assist as a supportive aid which can be used at the doctor's discretion in finally declaring a decision. A fuzzy clustering based segmentation of any brain MRI image has also been provided which can be used to study the intricate internal brain structure depending on the number of clusters chosen; hence it can be used as a study/analysis tool. The stage or grade of the tumor can also be calculated.

\section{BACKGROUND HISTORY}

Medical imaging has become the key to access inside human brain/head for the purpose of Diagnosis and treatment planning. In order to understand the effectiveness of planned treatment following the diagnosis, treated brain/head part may have to be monitored several times during a period of time. Information gained from successive imaging of brain/head part provides guidance to next step of treatment. Comparison of images or datasets taken at 
different times requires registration of these images or datasets since the same conditions may not be provided at all times. Accurate segmentation of the brain/head part under treatment is needed while comparing medical images to achieve quantitative and qualitative measurements. This segmentation task enables two dimensional and three dimensional visualizations of the region which also aid in directing the planning strategy. In past, several segmentation algorithms are investigated and a hybrid segmentation algorithm is developed in order to segment bone tissue out of head CT slices for orthodontic treatment planning. Using the developed segmentation algorithm, three dimensional visualizations of segmented bone tissue out of head CT slices of two patients are obtained. Visualizations are obtained using the MATLAB Computer software's visualization library. Besides these, methods are developed for automatic registration of two dimensional and threedimensional CT images taken at different time periods. These methods are applied to real and synthetic data. Algorithms and methods are implemented in MATLAB computer program. Medical Imaging gained an increased importance over the past thirty years after the invention of the computerized tomography and various researches have been carried out since then. The discovery of computerized tomography scan revolutionized medical diagnosis, allowing doctors to see exactly what is going on inside the brain/head. Combined with powerful, robust and fast segmentation, registration and visualization algorithms, it is now possible to carry out surgical planning even during the operation.

\section{EXISTING SYSTEM}

In, propose a hidden Andrei Markov random fieldExpectation Maximization (HMRF-EM) and threshold techniques for detection of brain tumor from MRI scan images. An image mining technique for brain tumor classification exploitation cropped association rule with MARI formula using association rule mining technique is proposed in. It combines the lowlevel options extracted from pictures and high level data from specialists. In planned early prediction of brain cancer supported texture options and neuro classification logic. Distinct options in conjunction with minimum distance area unit used for brain tumor detection in given MRI image. Extracted region is recognized exploitation neuro fuzzy approach. In designed of uncovering strategies to eliminate the non-brain tissue in resonance (MR) brain pictures. The pre-segmentation step is employed to seek out the optimum place to begin for the deformation and relies on thresholds and morphological operators. The deformable model relies on a simplex mesh and its deformation is controlled by the image native grey levels and also the data obtained on the grey level modeling of the presegmentation. Then the author tested Simplex Mesh and bar graph Analysis of uncovering (SMHASS) technique on the subsequent international databases unremarkably utilized in scientific articles: Brain Web, net Brain Segmentation Repository (IBSR), and Segmentation Validation Engine (SVE). During this paper a comparison was performed against 3 of the most effective uncovering strategies antecedently published: Brain Extraction Tool (BET), Brain Surface Extractor (BSE), and Hybrid Watershed formula (HWA). In the most technique used was segmentation that is completed employing technique supported threshold segmentation, watershed segmentation and morphological operators. In the planned segmentation technique was experimented with MRI scanned pictures of human brains: therefore locating tumor within the pictures. In conjointly mentioned future work is to increase their planned technique for color based mostly segmentation of 3D pictures. For this purpose would like a classification technique to prepare 3 dimensional objects into separate feature categories, whose characteristics will facilitate in identification 
of brain diseases. In evaluated nucleon man spectroscopy for brain tumor categorization. Brain tumors area unit classified into low-high grade interstitial tissue neoplasm, menengiomas and metastasis. Brain tumors area unit classified on the premise of Cho/NAA, Cho/Cr and Cho/MI substance ratios. In applied changed segmentation techniques on MRI scan pictures to observe brain tumor. Changed Probabilistic Neural Network supported Learning Vector division. With image and knowledge analysis to classify brain tumor exploitation MRI scans. In, the watershed and thresholding formula is evaluated for the detection and segmentation of brain tumor. Edge base segmentation and color base segmentation, Cohesion self-merging based mostly partition K-mean algorithm applied to extract the boundary and dimensions of the tumor. Morphological operation is applied to MRI pictures of brain. In [10], the image slicing exploitation bit plane technique and reconstructed exploitation Principal is used to extract the brain tumor. The dimensions of tumor and also the quantitative relation of tumor and non-tumor space is calculated using 2 by 2 neighborhood technique. In planned technique includes nonnative preprocessing, fuzzy intensification to reinforce the standard of the man pictures, $\mathrm{k}$ - suggests that bunch technique for brain tumor segmentation. The results area unit evaluated supported accuracy, sensitivity, specificity, false positive rate, false negative rate, Jaccard similarity metric and Dice constant. In order to observe this abnormal growth of tissue in a picture, in presents a unique theme that uses a ballroom dancing procedure; the $\mathrm{k}$-means technique and also the hierarchic center of mass form Descriptor (HCSD). The atomization of this method is beneficial for post process of the extracted region of interest just like the tumor segmentation. The bunch stage is applied to discriminate structures supported component intensity whereas the HCSD enable to pick out solely those having a selected form. A bounding box is then mechanically placed to delineate the region within which the tumor was found.

TABLE I

LIMITATION OF EXISTING SYSTEM

\begin{tabular}{|c|c|c|}
\hline \begin{tabular}{|l|} 
Sr. \\
No
\end{tabular} & $\begin{array}{l}\text { Proposed } \\
\text { Technique }\end{array}$ & Limitation \\
\hline 1. & $\begin{array}{l}\text { Fuzzy } \\
\text { models } \\
\text { Image } \\
\text { Fusion }\end{array}$ & $\begin{array}{l}\text { The proposed algorithm } \\
\text { consists of: the registration } \\
\text { of multispectral MR images, } \\
\text { the creation of fuzzy models } \\
\text { describing the } \\
\text { characteristics of tumor, the } \\
\text { fusion based on fuzzy fusion } \\
\text { operators and the } \\
\text { adjustment by fuzzy region } \\
\text { growing based on fuzzy } \\
\text { connecting. } \\
\text { Using linear image } \\
\text { registration tool for evaluate } \\
\text { the proposed method. }\end{array}$ \\
\hline 2. & $\begin{array}{l}\text { Histogram } \\
\text { - based. } \\
\text { Fuzzy C- } \\
\text { mean } \\
\text { smoothen } \\
\text { the } \\
\text { boundarie } \\
\text { s }\end{array}$ & $\begin{array}{l}\text { The FSL library tool based } \\
\text { software was compared with } \\
\text { the performance of the } \\
\text { proposed algorithm. It is } \\
\text { considered to be a good } \\
\text { candidate for fully } \\
\text { automatic MRI analysis } \\
\text { systems. } \\
\text { Because of the fully } \\
\text { automated nature of the } \\
\text { algorithm with no human } \\
\text { intervention, along with } \\
\text { lesser number of iterations } \\
\text { taken. }\end{array}$ \\
\hline 3. & $\begin{array}{c}\text { Fuzzy C- } \\
\text { Means }\end{array}$ & $\begin{array}{l}\text { - Generalized spatial fuzzy c- } \\
\text { means (CSFCM) algorithm } \\
\text { which possesses both pixel } \\
\text { attributes and spatial local } \\
\text { information that is weighted }\end{array}$ \\
\hline
\end{tabular}




\begin{tabular}{|c|c|c|}
\hline & & $\begin{array}{l}\text { in correspondence with } \\
\text { neighbor elements based on } \\
\text { their distance attributes. } \\
\text { - This has the potentiality to } \\
\text { improve the segmentation } \\
\text { performance tremendously. } \\
\text { This improves the } \\
\text { segmentation performance } \\
\text { dramatically. } \\
\text { Poor contrast, noise and } \\
\text { non-uniform intensity } \\
\text { variation can affect the } \\
\text { results }\end{array}$ \\
\hline 4. & $\begin{array}{c}\text { Fuzzy } \\
\text { possibilistic } \\
\text { C- means }\end{array}$ & $\begin{array}{l}\text { - The paper announce the } \\
\text { developed a hybrid } \\
\text { segmentation method that } \\
\text { uses both region and } \\
\text { boundary information of the } \\
\text { image to segment the tumor. } \\
\text { - Compare a fuzzy } \\
\text { classification method and a } \\
\text { symmetry analysis method } \\
\text { for detecting the tumors. } \\
\text { Evaluate the proposed } \\
\text { method by testing } 2 \text { images } \\
\text { provide by IBSR data. }\end{array}$ \\
\hline 5. & $\begin{array}{c}\text { Aggravatio } \\
\text { n of } \\
\text { filtering } \\
\text { method } \\
\text { Fuzzy } \\
\text { expert }\end{array}$ & $\begin{array}{l}\text { - Feature Extraction is done } \\
\text { by thresholding method. } \\
\text { And, they develop } \\
\text { Approximate Reasoning } \\
\text { method to recognize the } \\
\text { tumor grade in brain MRI. } \\
\text { - More than } 26.3 \% \text { of tested } \\
\text { image gives not correct } \\
\text { answer using both proposed } \\
\text { algorithm type I and type II. }\end{array}$ \\
\hline
\end{tabular}

\section{PROBLEM FORMULATION}

\section{PROBLEM DEFINITION}

- The most of existing methods has ignored the poor quality images like images with noise or poor brightness.

- Most of the existing researchers have neglected the use of object based segmentation; to detect tumors in brain.

\section{PROPOSED SYSTEM}

The CT scan images from normal subjects and patients with Brain/Head injury are acquired sing a data acquisition system as per standards of biomedical image processing. Programs are developed using MATLAB software package to analyze the digital stored data of CT scan and extract the features and brain/head injury parameters in normal subjects and patients using proposed techniques. Based on the quantitative assessment of parameter, an initial diagnostic evaluation can be carried out. These parameters can be fed Database model classifying normal subjects from brain/head injury patients.

\section{FLOW CHART}

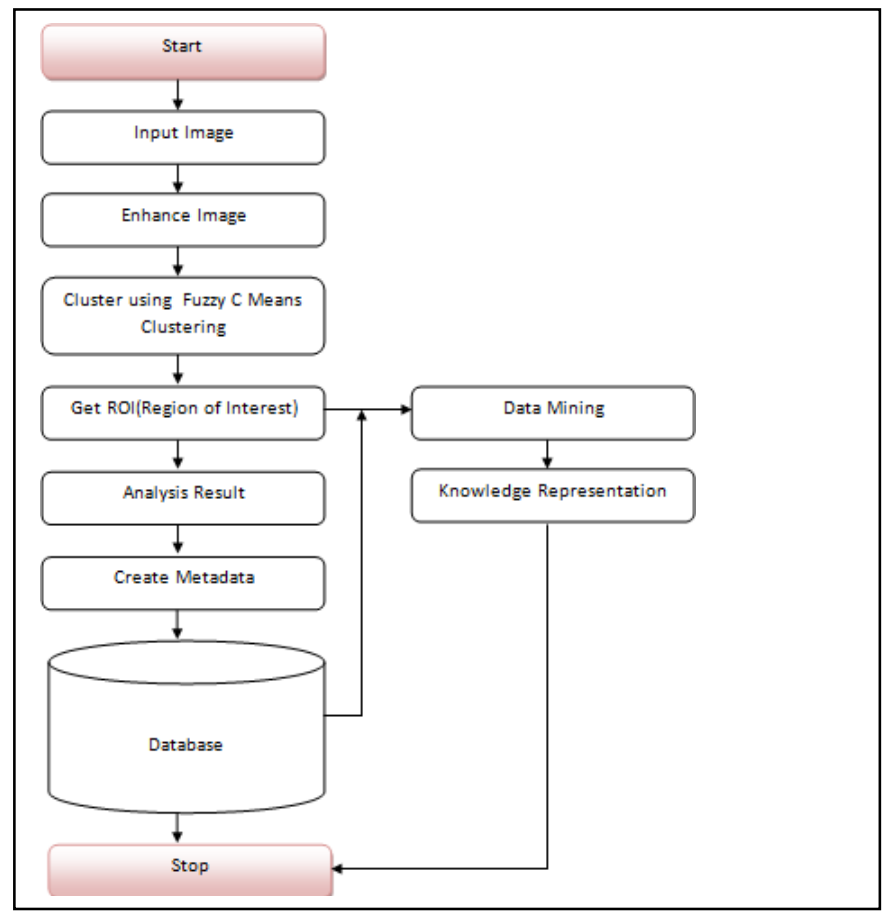

\section{DATA FLOW DIAGRAM}

3.1 Algorithm: 
I. Background Removal

1. Start

2. Input an Image (I)

3. For $\mathrm{i}=0$ to $\mathrm{W}(\mathrm{I})^{*} \mathrm{H}(\mathrm{I})$ Read

Pixel P(i) Extract RGB

If $(\mathrm{P}(\mathrm{i})>\& \& \mathrm{P}(\mathrm{i})<=30)$

If counter $=10$

Convert pixels $\mathrm{P}(\mathrm{i})$ to $\mathrm{P}(\mathrm{i}+10)$ to 255 End

4. Save Background Removed Image (Ig).

5. Stop

In training phase, concentration is on developing database that contains Images with tag information.

Input Image: An Input image is MRI Scan Image from an Authenticate source device

Enhance Image: Enhancing technique may be wavelet base enhance/histogram equalization base method

Clustering Method: Clustering will be done with CFuzzy Means / PSO/ SVM and with other methods for comparative analysis.

ROI: Region of an interest will be a step where actual region of an infection will be find out.

Metadata: Data about data is called as metadata. In this project, metadata will associate with Every ROI Pattern before loading it into database.

Data Mining: In this project, ROI Region will be match with database patterns and accordingly its knowledge will be presented.

\section{REFERENCES}

[1]. Rana Banik, Md. Rabiul Hasan, Md. Saif Iftekhar,"Automatic Detection, Extraction and Mapping of Brain Tumor from MRI Scanned Images using Frequency Emphasis Homomorphic and Cascaded Hybrid Filtering Techniques",2nd Int'l Conf. on Electrical Engineering and Infonnation \& Communication Technology (ICEEICT) 2015 Jahangirnagar
University, Dhaka-1342, Bangladesh, 21-23 May 2015

[2]. Hayder Saad Abdulbaqi, Mohd Zubir Mat Jafri, Ahmad Fairuz Omar, Kussay N. Mutter, Loay Kadom Abood, Iskandar Shahrim Bin Mustafa," Segmentation and Estimation of Brain Tumor Volume in Computed Tomography Scan Images Using Hidden Markov Random Field Expectation Maximization Algorithm", 2015 IEEE Student Conference on Research and Development (SCOReD, 978-1-4673-95724/15/\$31.00 (2015 IEEE.

[3]. Sajjad Mohsin, Sadaf Sajjad, Zeeshan Malik, and Abdul Hanan Abdullah," Efficient Way of Skull Stripping in MRI to Detect Brain Tumor by Applying Morphological Operations, after Detection of False Background", International Journal of Information and Education Technology, Vol. 2, No. 4, August 2012.

[4]. P.Rajendran and M.Madheswaran ,"An Improved Image Mining Technique For Brain Tumour Classification Using Efficient classifier",(IJCSIS) International Journal of Computer Science and Information Security,Vol. 6, No. 3, 2009.

[5]. G Vijay Kumar, Dr GV Raju, Biological Early Brain Cancer Detection Using Artificial Neural Network",IJCSE) International Journal on Computer Science and EngineeringVol. 02, No. 08, 2010, 2721-2725.

[6]. Francisco J. Galdamesa,d, Fabrice Jailletc,d, Claudio A. Pereza,b,"An Accurate Skull Stripping Method Based on Simplex Meshes and Histogram Analysis in Magnetic Resonance Images", Rapport de recherche RR-LIRIS-2011019.

[7]. Anam Mustaqeem, Ali Javed, Tehseen Fatima," An Efficient Brain Tumor Detection Algorithm Using Watershed \& Thresholding Based Segmentation", I.J. Image, Graphics and Signal 
Processing, 2012, 10, 34-39 Published Online September 2012 in MECS.

[8]. Meghana Nagori, Shivaji Mutkule, Praful Sonarkar ," Detection of Brain Tumor by Mining fMRI Images", International Journal of Advanced Research in Computer and Communication Engineering Vol. 2, Issue 4, January 2013.

[9]. Roshan G. Selkar Prof. M. N. Thakare ,"Brain Tumor Detection And Segmentation By Using Thresholding And Watershed Algorithm", Ijaict Volume 1, Issue 3, July 2014.

[10]. Madhav Kurupl, Abhijith Bailur2, Pavithra Rajeswaran2, Madhuneka Sundararajan2, Abhinav2," An innovative approach to monitor brain tumor propagation and track the efficacy of treatment by processing MR Images,"International Conference on Industrial Instrumentation and Control (ICIC),2015.

[11]. Mohan J, Krishnaveni V, Yanhui Huo Automated Brain Tumor Segmentation On MR Images Based On Neutrosophic Set Approach, IEEE Sponsored 2nd International Conference On Electronics And Communication System (ICECS 2015) 978-1-4788-7225- 8/15/\$31.00 (C)2015 IEEE 1078.

[12]. Elis'ee Ilunga-Mbuyamba1, Juan Gabriel AvinaCervantes1*, Dirk Lindner2, Jesus GuerreroTurrubiates1, Claire Chalopin3," Automatic Brain Tumor Tissue Detection based on Hierarchical Centroid Shape Descriptor in T1weighted MR images.”, International Conference On Electronics, Communications And Computers (Conielecomp) 2016.

\section{Cite this article as :}

Padmani S. Judape, Prof. Pragati Patil, Prof. Gajanan Patle, "Detection and Identification of Tumor Region from MRI Brain Image using Image Segmentation", International Journal of Scientific Research in Science and Technology (IJSRST), Online ISSN : 2395-602X, Print ISSN : 2395-6011, Volume 7 Issue 2, pp. 567-573, March-April 2020. Available at doi : https://doi.org/10.32628/IJSRST207283 Journal URL : http://ijsrst.com/IJSRST207283 